\title{
Analyse quantitative et qualitative de la microflore des poudres de lait écrémé
}

\author{
par \\ A. CHOPIN \\ Institut National de la Recherche Agronomique \\ Laboratoire de Recherches de Technologie Laitière \\ 65, rue de Saint-Brieuc - 35042 Rennes Cedex (France)
}

Actuellement, dans la pratique industrielle courante, le lait écrémé destiné à être concentré et séché sous forme de poudre subit, avant la concentration, un " préchauffage " à température élevée $\left(85^{\circ} \mathrm{C}\right.$ à $\left.95^{\circ} \mathrm{C}\right)$ pendant 10 à $20 \mathrm{~s}$ dans le but d'assurer une destruction suffisante de la flore microbienne. Bien que ce traitement thermique soit assez sévère, il conduit à une poudre dont la qualité bactériologique est parfois seulement moyenne.

De plus, cette technique présente certains inconvénients dont le plus grave est d'entraîner la dénaturation des protéines solubles du lait. Si cette dénaturation n'a que peu de répercussions sur la valeur nutritionnelle du produit (Adrian, 1975), elle risque cependant de conduire au déclassement technique de la poudre de lait. En effet, la tendance de nombreux utilisateurs de lait en poudre est de préférer les poudres n'ayant subi qu'un chauffage modéré ou faible (poudres désignées dans la terminologie anglo-saxonne par les noms " mediumheat » ou " low-heat »). Ces poudres sont définies par une teneur en protéines solubles mesurée dans des conditions normalisées (ADMI, 1965). Les poudres « low-heat " doivent contenir plus de $6 \mathrm{mg} / \mathrm{g}$ d'azote provenant des protéines solubles tandis que les poudres « medium-heat » doivent en contenir une quantité comprise entre 1,5 et $6 \mathrm{mg} / \mathrm{g}$. D'après O'Connor (1969), un préchauffage plus sévère que $72^{\circ} \mathrm{C}$ pendant $30 \mathrm{~s}$ ne permet pas d'obtenir de poudre « low-heat».

Il serait donc intéressant d'abaisser les températures de préchauffage utilisées actuellement en recherchant d'autres moyens de contrôler le nombre de bactéries survivant dans la poudre. Auparavant, il nous a semblé nécessaire de préciser la nature de la flore microbienne dominante présente dans les poudres de lait fabriquées en France. A titre de comparaison, quelques échantillons produits dans d'autres pays que la France ont également été étudiés. 


\section{MATERIEL ET METHODES}

\section{Origine des échantillons de poudre de lait écrémé}

Cent-cinquante et un échantillons de poudre de lait écrémé, destinée soit à la consommation humaine, soit à la consommation animale, ont été obtenus de sept installations industrielles de séchage situées dans l'Ouest de la France. La production de chacune de ces usines variait entre 1200 t et 25000 t de poudre de lait écrémé par an. Notre échantillonnage, effectué de novembre 1973 à novembre 1974 , a porté approximativement sur le $1 / 10$ de la production nationale française (cette dernière atteignant environ $600000 \mathrm{t}$ par an).

Vingt-neuf échantillons de poudre, dont une poudre «low-heat », provenaient de sept installations industrielles fonctionnant à l'étranger. Les prélèvements se sont échelonnés de janvier à mars 1975.

\section{Dénombrement de la flore totale}

La reconstitution des échantillons et le dénombrement de la flore totale ont été effectués conformément à la norme de la Fédération Internationale de Laiterie (F.I.L.I.D.F. 49 : 1970). Cette méthode consiste à reconstituer l'échantillon à $47 \pm 2^{\circ} \mathrm{C}$ et à dénombrer sa flore totale en $72 \mathrm{~h}$ d'incubation à $30^{\circ} \mathrm{C}$ avec le milieu Plate Count Agar additionné de 1 p. 1000 de lait en poudre.

\section{Détermination de la flore dominante}

Pour chaque échantillon, trois à cinq colonies isolées, prélevées au hasard sur la gélose nutritive étaient purifiées et identifiées. Toutes les colonies isolées ont pu être rattachées à l'un des groupes suivants :

- Microbacterium : identifié suivant Orla-Jensen (1919), Robinson (1966) et Bergey (1974). On a regroupé dans ce genre de petites bactéries immobiles, Gram positives, à catalase positive, non sporulées, très thermorésistantes, formant à la surface des milieux gélosés des colonies de couleur jaune pâle à jaune vif.

- Bacillus : on a regroupé dans ce genre les bactéries sporulées à catalase positive.

- Streptococcus : on a regroupé dans ce genre les cocci à catalase négative. Ces germes étaient tous aptes (à l'exception d'une souche de Str. Lactis) à se développer à $45^{\circ} \mathrm{C}$. Ils ont été répartis en deux groupes : streptocoques du groupe viridans (Str. thermophilus, Str. uberis) et streptocoques du groupe faecalis selon qu'ils étaient aptes ou non à se développer à $10^{\circ} \mathrm{C}$, en présence de $6,5 \mathrm{p} .100 \mathrm{de}$ $\mathrm{NaCl}$, à réduire le lait tournesolé et le lait additionné de 0,1 p. 100 de bleu de méthylène. 
- Micrococcus et Staphylococcus : on a regroupé ici les cocci à catalase positive. La différenciation entre Micrococcus et Staphylococcus portait sur leur aptitude à fermenter le glucose en anaérobiose (Abd-El-Malek et Gibson, 1948). Dans le genre Staphylococcus ainsi défini, la distinction entre espèces et souches pathogènes et espèces saprophytes n'a pas été faite.

\section{RESULTATS}

\section{Dénombrement de la flore totale}

Pour les poudres fabriquées en France, le nombre de germes par gramme variait entre 160 et 1400000 avec une moyenne de 9100 et une médiane (1) de 9400 . Pour les poudres fabriquées à l'étranger, ces chiffres variaient entre 3000 et 440000 avec une moyenne de 8000 et une médiane de 5900 . La poudre "low-heat " analysée contenait 440000 germes/g.

\section{Identification de la flore dominante}

Pour l'étude de la nature de la flore dominante, 760 souches bactériennes ont été isolées (616 souches des poudres d'origine française et 144 souches des poudres d'origine étrangère) et identifiées.

Les résultats obtenus montrent que la flore des poudres de lait écrémé fabriquées en France (tab. 1) se compose par ordre d'importance décroissante de bactéries appartenant aux genres Microbacterium (64 p. 100), Bacillus (23 p. 100), Streptococcus (9,5 p. 100) (streptocoques du groupe viridans : 6,5 p. 100 , streptocoques du groupe D : 3 p. 100), Micrococcus (2 p. 100) et Staphylococcus (1,5 p. 100).

La flore des poudres de lait fabriquées à l'étranger (tab. 2) se compose de bactéries appartenant aux genres Bacillus (74 p. 100), Streptococcus (18 p. 100) et Microbacterium ( 8 p. 100).

On observe (tab. 1) que cette répartition, pour les poudres fabriquées en France, varie beaucoup avec le nombre de germes présents dans l'échantillon. La flore dominante des poudres à faible teneur en micro-organismes se compose essentiellement de bactéries appartenant au genre Bacillus. Les poudres dont la teneur en microorganismes varie de moyenne à forte, renferment surtout des bactéries appartenant au genre Microbacterium. Les streptocoques constituent la flore dominante des poudres à teneur élevée en microorganismes. Le genre Micrococcus est rencontré uniformément quel que soit le nombre de germes/g. Le genre Staphylococcus est surtout trouvé dans les poudres à teneur moyenne à faible en micro-organismes.

(1) 50 p. 100 des poudres contenaient un nombre de germes/g inférieur à la médiane, 50 p. 100 contenaient un nombre de germes/g supérieur. 
TABLEAU 1. - Répartition des genres bactériens dans les poudres de lait écrémé fabriquées en France en fonction du nombre de germes par gramme de poudre

\begin{tabular}{|c|c|c|c|c|c|c|c|c|}
\hline $\begin{array}{l}\text { Nombre de } \\
\text { germes/g de poudre }\end{array}$ & $\begin{array}{l}\text { Nombre } \\
\text { de souches } \\
\text { identifiées }\end{array}$ & $\begin{array}{c}\text { Micro- } \\
\text { bacterium }\end{array}$ & Bacillus & $\begin{array}{l}\text { Strepto- } \\
\text { coccus } V^{* *}\end{array}$ & $\begin{array}{c}\text { Strepto- } \\
\text { coccus } D^{* * * *}\end{array}$ & $\left|\begin{array}{c}\text { Strepto- } \\
\text { coccus lactis }\end{array}\right|$ & $\begin{array}{l}\text { Micro- } \\
\text { coccus }\end{array}$ & $\begin{array}{l}\text { Staphylo- } \\
\text { coccus }\end{array}$ \\
\hline$<8,0.10^{2}$ & 16 & $1(6)^{*}$ & $13 \quad(82)$ & 0 & 0 & 0 & $1(6)$ & 1 (6) \\
\hline $8,0.10^{2}$ à $1,6.10^{3}$ & 97 & $27 \quad(28)$ & $60 \quad(62)$ & 0 & $9 \quad(9)$ & 0 & 0 & 1 (1) \\
\hline $1,7.10^{3}$ à $3,6.10^{3}$ & 88 & $41 \quad(47)$ & $41 \quad(47)$ & 0 & $2 \quad(2)$ & 0 & $2(2)$ & 2 (2) \\
\hline $3,7 \cdot 10^{3}$ à $7,9.10^{3}$ & 87 & $70 \quad(80)$ & $14 \quad(16)$ & 0 & 0 & 0 & 0 & 3 (4) \\
\hline $8,0.10^{3}$ à $1,6.10^{4}$ & 103 & $92 \quad(89)$ & $7 \quad(7)$ & 0 & 0 & 0 & 2 (2) & $2(2)$ \\
\hline $1,7 \cdot 10^{4}$ à $3,6 \cdot 10^{4}$ & 79 & $67 \quad(85)$ & $4 \quad(5)$ & 0 & $3 \quad(4)$ & 0 & $5 \quad(6)$ & 0 \\
\hline $3,7.10^{4}$ à $7,9.10^{4}$ & 71 & $63 \quad(89)$ & $1 \quad(1)$ & $2 \quad(3)$ & $3 \quad(4)$ & 0 & $2(3)$ & 0 \\
\hline $8,0.10^{4}$ à $1,6.10^{5}$ & 17 & $13 \quad(76)$ & 0 & $2(12)$ & $2(12)$ & 0 & 0 & 0 \\
\hline $1,7.10^{5}$ à $3,6 \cdot 10^{5}$ & 38 & 17 (44) & 0 & $19 \quad(50)$ & 0 & 1 (3) & 1 (3) & 0 \\
\hline $3,7 \cdot 10^{5}$ à $7,9 \cdot 10^{5}$ & 17 & $3(18)$ & 0 & $14 \quad(82)$ & 0 & 0 & 0 & 0 \\
\hline$>8,0.10^{5}$ & 3 & 0 & 0 & $3(100)$ & 0 & 0 & 0 & 0 \\
\hline Total & 616 & $394 \quad(64)$ & $140 \quad(23)$ & $40(6,5)$ & $19(3,1)$ & $1(0,2)$ & $13(2,1)$ & $9(1,5)$ \\
\hline
\end{tabular}

* Le premier chiffre indique le nombre de souches isolées pour chacun des genres microbiens étudiés. Le chiffre entre paren. thèses indique le pourcentage que représentent ces souches par rapport au nombre correspondant de souches identifiées.

** Streptococcus du groupe viridans (Str. thermophilus et Str. uberis).

*** Streptococcus du groupe sérologique D (Str. faecalis, Str. faecium/durans). 
TABLEAU 2. - Répartition des genres bactériens dans des poudres de lait écrémé d'origine étrangère en fonction du nombre de germes par gramme de poudre

\begin{tabular}{|c|c|c|c|c|}
\hline Nombre de germes & $\begin{array}{c}\text { Nombre de souches } \\
\text { identifiées }\end{array}$ & Microbacterium & Bacillus & Streptococcus \\
\hline$<8,0.10^{2}$ & 0 & $0 \quad$ * & 0 & 0 \\
\hline $8,0.10^{2}$ à $1,6.10^{3}$ & 0 & 0 & 0 & 0 \\
\hline $1,7.10^{3}$ à $3,6.10^{3}$ & 14 & $2(14)$ & $10 \quad(71)$ & $2(15)$ \\
\hline $3,7 \cdot 10^{3}$ à $7,9 \cdot 10^{3}$ & 95 & $6 \quad(6)$ & $86 \quad(91)$ & $3 \quad(3)$ \\
\hline $8,0.10^{3}$ à $1,6.10^{4}$ & 20 & 3 (15) & $9 \quad(45)$ & $8 \quad(40)$ \\
\hline $1,7 \cdot 10^{4}$ à $3,6 \cdot 10^{4}$ & 0 & 0 & 0 & 0 \\
\hline $3,7 \cdot 10^{4}$ à $7,9 \cdot 10^{4}$ & 5 & 0 & 0 & $5(100)$ \\
\hline $8,0.10^{4}$ à $1,6.10^{5}$ & 5 & 0 & 0 & $5(100)$ \\
\hline $1,7.10^{5}$ à $3,6.10^{5}$ & 0 & 0 & 0 & 0 \\
\hline $3,7.10^{5}$ à $7,9.10^{5}$ & 5 & $1 \quad(20)$ & $1 \quad(20)$ & $3(60)$ \\
\hline$>8,0.10^{5}$ & 0 & 0 & 0 & 0 \\
\hline Total & 144 & $12 \quad(8)$ & $106 \quad(74)$ & $26 \quad(18)$ \\
\hline
\end{tabular}

* Le premier chiffre indique le nombre de souches isolées pour chacun des genres microbiens étudiés. Le chiffre entre parenthèses indique le pourcentage que représentent ces souches par rapport au nombre correspondant de souches identifiées. 
Cette répartition ne dépend apparemment que du nombre de germes présents dans l'échantillon. En particulier pour des nombres de germes du même ordre, aucune différence dans la nature de ces germes n'apparaît en fonction de l'installation de séchage dont provient l'échantillon.

\section{Thermorésistance et températures de croissance des Microbacterium}

La fréquence des bactéries appartenant au genre Microbacterium dans les poudres de lait nous a conduit à étudier plus en détail leurs températures de croissance et leurs possibilités de thermorésistance, caractéristiques importantes permettant d'expliquer la présence éventuelle en grand nombre de ces germes dans les poudres de lait.

La thermorésistance a été mesurée conformément à la méthode décrite par Robinson (1966). Cette méthode consiste à chauffer, pendant $10 \mathrm{mn}$, en ampoules scellées, à des températures allant de $65^{\circ} \mathrm{C}$ à $85^{\circ} \mathrm{C}$, un bouillon nutritif ensemencé en Microbacterium. Après chauffage, l'incubation des ampoules permet d'apprécier la survie de la souche étudiée. Les résultats obtenus sont présentés dans le tableau 3. La distribution de ce caractère de thermorésistance des

\section{TABLEAU 3}

Thermorésistance des Microbacterium isolés des poudres de lait

\begin{tabular}{c|c|c}
\hline Chauffage & Nombre résistants & p. 100 résistants \\
\cline { 2 - 3 } $85^{\circ} \mathrm{C}, 10 \mathrm{mn}$ & 25 & 6,3 \\
$80^{\circ} \mathrm{C}, 10 \mathrm{mn}$ & 180 & 45,7 \\
$75^{\circ} \mathrm{C}, 10 \mathrm{mn}$ & 335 & 85,0 \\
$70^{\circ} \mathrm{C}, 10 \mathrm{mn}$ & 380 & 96,4 \\
$65^{\circ} \mathrm{C}, 10 \mathrm{mn}$ & 394 & 100,0 \\
& & \\
\hline
\end{tabular}

souches de Microbacterium n'a pu être reliée ni à l'importance de la flore totale présente dans la poudre dont elles ont été isolées, ni à l'installation de séchage dont elles proviennent.

La croissance a été estimée en notant l'apparition d'un trouble visible dans un bouillon nutritif (tryptone Difco 1 p.100, peptone 
Evans 0,5 p. 100, Lab-Lemco 0,4 p. 100, lactose 0,3 p. 100, Delpui, 1969) en $48 \mathrm{~h}$ à $37^{\circ} \mathrm{C}, 72 \mathrm{~h}$ à $30^{\circ} \mathrm{C}$, et $7 \mathrm{j}$ à $10^{\circ} \mathrm{C}$. Toutes les souches se développent à $30^{\circ} \mathrm{C}, 79 \mathrm{p} .100$ se multiplient à $37^{\circ} \mathrm{C}$ et $79 \mathrm{p} .100$ également à $10^{\circ} \mathrm{C}$.

\section{DISCUSSION}

Si l'on compare nos résultats concernant le dénombrement de la flore totale des poudres de lait écrémé fabriquées en France à la norme française correspondant au lait en poudre destiné à la consommation humaine directe (moins de 50000 bactéries aérobies revivifiables dans $1 \mathrm{~g}$ ), on constate que 84 p. 100 des échantillons faisant partie de notre étude satisfont à ladite norme. Ce pourcentage d'échantillons satisfaisants passe à 94 p. 100 si l'on considère la norme correspondant à la poudre de lait destinée aux utilisations industrielles (biscuiterie, etc.) (moins de 200000 bactéries aérobies revivifiables dans $1 \mathrm{~g}$ ). Il n'y a pas de norme bactériologique officielle pour la poudre de lait destinée à l'alimentation du bétail qui représente cependant plus de la moitié de la poudre produite en France.

On peut aussi comparer ces chiffres à ceux qui ont été obtenus dans différents pays, à différentes époques. En 1954, aux Pays-Bas, (Galesloot, 1955) 95 p. 100 des poudres de lait écrémé contenaient moins de 250000 germes par gramme. En 1966, dans ce même pays (Galesloot, 1968) 94 p. 100 des poudres de lait contenaient moins de 50000 germes par gramme. Aux Etats-Unis, Hall (1967) a montré que 85 p. 100 des poudres de grade A analysées contenaient moins de 30000 germes par gramme.

Les poudres de lait françaises étudiées contiennent donc des nombres de germes par gramme comparables à ceux que l'on peut observer dans d'autres pays à industrie laitière développée.

Il semble par contre que la nature des germes que nous avons rencontrés soit assez différente. Galesloot (1968) a trouvé que le genre microbien dominant dans les poudres de lait fabriquées aux Pays-Bas était le genre Streptococcus. Ce résultat a été confirmé par Naguib (1972) en Egypte. Les poudres originaires de l'étranger que nous avons analysées contiennent essentiellement des Bacillus, genre également rencontré en quantités importantes par Galesloot (1968) dans les poudres fabriquées aux Pays-Bas. Nos résultats montrent que ces deux genres sont assez peu représentés dans les poudres fabriquées en France où domine largement au contraire le genre Microbacterium. Ce résultat est à rapprocher de ceux de Mattick, Hiscox et Crossley (1945) qui ont également décrit des Microbacterium dans la flore dominante des poudres produites en Grande-Bretagne.

Pour expliquer la présence d'un grand nombre de bactéries dans la poudre de lait, deux hypothèses peuvent être retenues. Ou bien le 
lait utilisé pour fabriquer la poudre contient un grand nombre de bactéries thermorésistantes insuffisamment détruites par le préchauffage, ou bien les bactéries présentes dans le lait, ou apportées par une contamination extérieure, ont pu se multiplier au cours de la concentration du lait ou de la conservation du lait concentré juste avant séchage.

En ce qui concerne les Microbacterium, Robinson (1966), étudiant leurs températures de croissance, a montré qu'ils se multiplaient bien entre $30^{\circ} \mathrm{C}$ et $37^{\circ} \mathrm{C}$, mais qu'à $39^{\circ} \mathrm{C}$ seules $20 \mathrm{p} .100$ des souches se multipliaient encore. Nous avons montré que 21 p. 100 des 394 souches de Microbacterium isolées des poudres ne se multipliaient plus à $37^{\circ} \mathrm{C}$. De plus, même à la température optimum de croissance, leur multiplication demeure lente dans le lait. Il faut donc admettre que ces bactéries se trouvent présentes dans le lait de départ et ne sont pas détruites aux températures de préchauffage utilisées. Cette hypothèse est confirmée par l'observation que nous avons faite de leur thermorésistance extrêmement marquée.

Dans le cas où l'on emploie des températures de préchauffage très élevées $\left(92^{\circ} \mathrm{C}-95^{\circ} \mathrm{C}\right)$, on observe que seules survivent les spores bactériennes du lait que l'on retrouve alors en flore dominante, c'est bien ce que nous-mêmes avons observé avec les poudres à faible teneur en micro-organismes.

Par contre, il semble exclu que des bactéries appartenant au genre Streptococcus puissent survivre en nombres élevés aux températures de préchauffage pratiquées couramment. Néanmoins, dans des conditions de fabrication défecteuses, une multiplication microbienne peut avoir lieu (Crossley, 1942), si le nettoyage du concentrateur n'est pas satisfaisant ou si la concentration est poursuivie pendant plus de $8 \mathrm{~h}$ sans nettoyage de l'installation (Crossley, 1944 1945). Il a été montré de plus (Galesloot, 1968) que la concentration $\mathrm{du}$ lait en matière sèche favorise le développement de certaines bactéries, en particulier les streptocoques du groupe D.

La présence de Streptococcus dominants dans les poudres de lait renfermant des nombres très élevés de germes par gramme est donc à attribuer à des conditions de fabrication inadéquates permettant le développement de ces bactéries dans le lait au cours de la concentration et/ou de la conservation du lait concentré avant séchage.

A part ce cas, auquel il est possible de remédier assez facilement, il apparaît que les teneurs élevés en bactéries dans le lait en poudre sont attribuables à la présence dans le lait de départ d'un nombre relativement grand de bactéries thermorésistantes appartenant au genre Microbacterium. Seules des températures de préchauffage élevées permettent, dans l'état actuel de la technologie, d'éliminer ces germes. 


\section{Remerciements}

Nous remercions vivement tous les responsables d'installations de séchage qui ont accepté de nous faire parvenir des échantillons de leur production de poudre de lait.

Nous remercions également MM. Mocquot et Hermier dont les suggestions et les critiques nous ont été précieuses dans la présentation de ce travail.

\section{Rés u m é}

On a déterminé le nombre de germes présents dans 180 échantillons de poudres de lait écrémé. 151 échantillons avaient été fabriqués en France, 29 à l'étranger. Les moyennes sont respectivement 9100 et 8000 germes $/ \mathrm{g}$. Les résultats obtenus sur les poudres fabriquées en France, plus nombreux, sont également plus dispersés.

La nature de la flore microbienne présente dans ces échantillons a été étudiée en isolant et identifiant 760 souches bactériennes. Les poudres fabriquées à l'étranger contiennent surtout des Bacillus (74 p. 100 des souches isolées) tandis que les poudres fabriquées en France contiennent surtout des Microbacterium (64 p. 100 des souches isolées). Ces bactéries, les plus thermorésistantes des bactéries non sporulées, présentes dans le lait destiné à être séché, ne peuvent être éliminées que par un préchauffage à température élevée.

\section{S u $\mathrm{m}$ m a r y}

The bacterial plate counts of 180 samples of skim milk powder were determined. 151 samples were from french origin, 29 from foreign origin. The averages were respectively 9100 and 8000 microorganisms/g. Bacterial counts of french skim milk powders, more numerous, were also more dispersed.

760 bacterial strains were isolated and identified to study the nature of the microflora encountered in these samples. 74 p. 100 of the strains isolated from skim milk powders of foreign origin belong to the genus Bacillus. 64 p. 100 of the strains isolated from samples of french origin belong to the genus Microbacterium. This genus is one of the most thermoresistant among non sporulating genera. When these organisms are present in a milk to be spray-dried, they can only be destroyed by a high preheating temperature. 


\section{Références bibliographiques}

Abd-el-Malek (Y.) and Gibson (T.) (1948). - Studies in the bacteriology of milk. II. The staphylococci and microcci of milk. J. Dairy Res., 15, 249-260.

ADRIAN (J.) (1975). - Les traitements thermiques appliqués aux produits laitiers et leurs conséquences dans le domaine azoté. Le Lait, 55, 24-40, 182-206.

AMERICAN DRy Milk Institute (1965). - Standards for Grades for the Dry Milk Industry. Bull. 916, Chicago, 42 p.

Buchanan (R. E.), Gibbons (N. E.) (1974). - Bergey's Manual. Eight Edition, Baltimore, Williams and Wilkins.

Crossley (E. L.), Johnson (W. A.) (1942). - Bacteriological aspects of the manufacture of spray-dried milk and whey powders, including some observations concerning moisture content and solubility. J. Dairy Res., 13, 5-44.

Delpui (G.) (1969). - Communication personnelle.

F.I.L.-I.D.F. (1970). - Méthode normalisée pour le dénombrement des germes totaux dans les poudres de lait et de lactosérum (méthode de référence), $\mathrm{n}^{\circ} 49$.

GalesLoot (T. E.) (1955). - Over de oorzaak van verhogingen van het kiemgetal van verstuivingsmelkpoeder. Neth. Milk Dairy J., 9, 194-203.

Galesloot (T. E.), StadHouders (J.) (1968). - The microbiology of spray-dried milk products with special reference to Staphylococcus aureus and Salmonella. Neth. Milk Dairy J., 22, 158-172.

Hall (H. E.), Brown (D. F.), Robinson (H. M.), Donnelly (C. B.), Reyes (A. L.) (1967). - Bacteriological examination of grade A milk powder. J. Milk Food Technol., 30, 219-221.

Mattick (A.T.R.), Hiscox (E. R.), CRossley (E. L.) (1944-1945). - The effect of the various factors upon the bacterial plate count of the intermediate products and of the final powder. J. Dairy Res., 14, 135-144.

Naguib (E.), Abd-el-Ghani (S.), Taha (S. M.) (1972). - Spray-dried milk of the Sakha plant. II. Identification of predominating microorganisms. J. Milk Food Technol., 35 (1), 45-48.

O'Connor (F.), Mc Kenna (B. M.), O'Sullivan (A. C.) (1969). - Bacteriological and whey protein denaturation aspects of heating processes used in the manufacture of low-heat skimmilk powder. Ir. J. Agric. Res., 8, 417-430.

Orla-Jensen (S.) (1919). - The lactic acid bacteria. Copenhagen, Andr. Fred Horst and Son.

Robinson (K.) (1966). - Some observations on the taxonomy of the genus Microbacterium. I. Cultural and physiological reactions and heat resistance. J. Appl. Bact., 29, 607-615. 